\title{
Communications
}

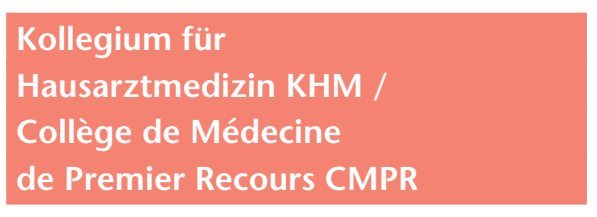

Daten der Kurse für den Erwerb des Fähigkeitsausweises Praxislabor (FAPL) 2010-2011

Dates des Cours AFCLP «Attestation de formation complémentaire du laboratoire du praticien» 2010-2011

Kurse über 3 Tage auf Deutsch in Luzern: 20.-22. Oktober 2010, Kurs LU-2010_02 8.-11. Februar 2011, Kurs LU-2011_01 13.-15. April 2011, Kurs LU-2011_02

Wichtig: Präferenz wird Kandidatinnen und Kandidaten gegeben, die kurz vor der Praxiseröffnung stehen.

Vereinbaren Sie mit dem Sekretariat KHM Ihr gewünschtes Kursdatum: KHM, Landhausweg 26, 3007 Bern, E-Mail: khm@hin.ch, Tel. 03137006 70. Sie erhalten anschliessend ein Anmeldeformular samt Rechnung für Kurs und FKA und werden zum gewählten Kurs eingeladen. Nach erfolgreichem Kursbesuch erhalten Sie den Fähigkeitsausweis mit Urkunde (vgl. auch www.kollegium.ch/aus/pld.html).

Sont prévus les cours suivants en langue française (durée 3 jours) à Lausanne:

Juin 23-25 2010, Cours LS 1/10

2011, Dates en préparation

Important: La préférence sera donnée aux candidat(e)s proches de leur installation.

Inscription: Négotiez d'abord une date convenable avec le secrétariat du Collège de Médecine de Premier Recours CMPR, Landhausweg 26, 3007 Bern, e-Mail: khm@hin.ch, tél. 0313700670 . Vous recevrez ensuite un formulaire d'inscription et un bulletin de versement pour le cours choisi et le certificat AFCLP. Voir aussi www.kollegium.ch/aus/ plf.html

\section{Société Suisse de Gastroentérologie}

\section{Prix et Bourses 2010}

La Société suisse de Gastroentérologie (SGG/ SSG), la Société suisse de Chirurgie viscérale (SGVC-SSCV), l'Association suisse pour l'étude du foie (SASL) ainsi que la Société suisse de Nutrition clinique (SSNC) décernent les prix et bourses suivants lors du congrès annuel 2010:

- Prix d'honneur de Fr. 15000 .-, décerné par la Société suisse de Gastroentérologie

- Prix d'Hépatologie Senior de Fr. 12500.-, décerné par la Maison Essex Chemie

- Prix d'Hépatologie Junior de Fr. 7500.-, décerné par la maison Roche Pharma

- Prix de Nutrition clinique de Fr. 10000.-, décerné par la maison Nycomed Pharma

- Prix Junior IBD de Fr. 5000.-, décerné par la Maison Essex Chemie

- Prix d'encouragement de Fr. 7500.-, décerné par la Maison AstraZeneca

- Prix Poster de Nutrition de Fr. 3000.-, décerné(s) par la Maison Fresenius Kabi SA

- 3 Prix Posters (3 abonnements), décernés par la Maison S. Karger

Les demandes doivent se faire électroniquement d'ici au 31 mai 2010: www.sgg-sgvc-congress.ch/ prizes.php.

Les informations et le règlement se trouvent sur www.sggssg.ch et www.sgg-sgvc-congress. ch/prizes.php

\section{Société Suisse \\ de Médecine Intensive SSMI}

\section{Prix SSMI 2010}

Dans le cadre de la Réunion annuelle de la Société Suisse de Médecine Intensive, deux prix d'un montant total de Fr. 8000.- seront attribués pour les meilleurs travails scientifiques publiés dans le domaine de la Médecine Intensive. Ces prix concernent les travaux publiés ou acceptés pour publication en 2009/2010 dans des revues révisées. Ceux-ci doivent provenir d'une unité de soins intensifs suisse. Plus d'informations au sujet du prix SSMI et les lauréats vous trouvez sur le site de l'association www.sgi-ssmi.ch dans la rubrique recherche.

Les manuscrits sont à adresser au président de la SSMI au plus tard jusqu'au 30 avril 2010: sgi@imk.ch, Pr H. Pargger, Président SSMI, Schweizerische Gesellschaft für Intensivmedizin (SGI), c/o IMK, Münsterberg 1, 4001 Bâle. Les prix seront remis aux bénéficiaires lors de la réunion annuelle du 2 au 4 septembre 2010 à Lausanne.

\section{Examen de spécialiste}

\section{Examen de spécialiste en vue}

de l'obtention du titre de spécialiste en angiologie

\section{Première partie (examen écrit)}

Lundi, 13 septembre 2010, lors du $15^{\mathrm{e}}$ Congrès Tripartite des Sociétés Suisse, Allemande et Autrichienne d'Angiologie et du 11 $1^{\mathrm{e}}$ Congrès de l'Union des Sociétés Suisses des Maladies Vasculaires à Bâle

Deuxième partie (examen oral et pratique)

Vendredi, 29 octobre 2010 à l'Hôpital de l'Ile, Berne

Délai d'inscription: le $1^{\text {er }}$ août 2010

Vous trouverez de plus amples informations sur le site web de l'ISFM www.siwf.ch $\rightarrow$ Formation postgraduée médecins-assistants $\rightarrow$ Examen de spécialiste 